\title{
miR-10a in Peripheral Blood Mononuclear Cells Is a Biomarker for Sepsis and Has Anti-Inflammatory Function
}

\author{
Guoping Zheng, ${ }^{1}$ Guanguan Qiu, ${ }^{1}$ Menghua Ge, ${ }^{1}$ Jianbiao Meng ${ }^{\circ},{ }^{2}$ Geng Zhang, ${ }^{2}$ \\ Jiangmei Wang, ${ }^{3}$ Ruoqiong Huang, ${ }^{3}$ Qiang Shu $\mathbb{D}^{3},{ }^{3}$ and Jianguo Xu $\mathbb{D}^{1,3}$ \\ ${ }^{1}$ Shaoxing Second Hospital, 123 Yanan Road, Shaoxing, Zhejiang 312000, China \\ ${ }^{2}$ Tongde Hospital of Zhejiang, 132 Tianmushan Road, Hangzhou, Zhejiang 310007, China \\ ${ }^{3}$ Children's Hospital, Zhejiang University School of Medicine, National Clinical Research Center for Child Health, \\ 3333 Binsheng Road, Hangzhou, Zhejiang 310052, China
}

Correspondence should be addressed to Jianguo Xu; jxu5@yahoo.com

Received 18 September 2019; Accepted 30 December 2019; Published 22 January 2020

Academic Editor: Oleh Andrukhov

Copyright $\odot 2020$ Guoping Zheng et al. This is an open access article distributed under the Creative Commons Attribution License, which permits unrestricted use, distribution, and reproduction in any medium, provided the original work is properly cited.

Background. Recent literature has reported the use of circulating microRNAs (miRNAs) as biomarkers for sepsis. Immune cells play an essential role in the pathophysiology of sepsis. The aim of this prospective study was to identify miRNAs in peripheral blood mononuclear cells (PBMC) that could differentiate between sepsis and infection based on Sepsis-3 definition. Methods. A total of 62 patients (41 with sepsis and 21 with infection suffering from pneumonia but without sepsis) and 20 healthy controls were enrolled into the study. PBMC at admission were examined for a panel of 4 miRNAs (miR-10a, miR-17, miR-27a, and miR125b), which have been documented to participate in inflammatory response in immune cells, via qRT-PCR. Data were validated in a mouse model of sepsis induced via cecal ligation and puncture (CLP) and THP-1 monocytes. Results. miR-10a levels in PBMC at admission were significantly lower in sepsis patients compared with patients with infection and healthy controls. miR-10a levels were negatively correlated with disease severity scores as well as levels for c-reactive protein and procalcitonin. In addition, low miR-10a expression had a diagnostic value for sepsis and a prognostic value for 28-day mortality in receiving operating characteristic analysis. Compared with infection patients and healthy controls, PBMC from sepsis patients also had higher levels of mitogen-activated kinase kinase kinase 7 (MAP3K7), a known target protein of miR-10a and an activator of the NF- $\kappa \mathrm{B}$ pathway. In the mouse model of CLP-induced sepsis, miR-10a levels in PBMC were significantly decreased as early as $8 \mathrm{~h}$ after CLP. Overexpression of miR-10a in THP-1 cells significantly reduced the expression of MAP3K7 and proinflammatory cytokines including IL-6, TNF- $\alpha$, and MCP-1. Conclusions. PBMC miR-10a levels are decreased in sepsis and negatively correlated with the disease severity. Levels of miR-10a could distinguish between sepsis and infection and predict 28-day mortality. miR-10a plays an anti-inflammatory role in the pathogenesis of sepsis.

\section{Background}

Sepsis is currently defined as a life-threatening organ dysfunction caused by an infection with an increase in the Sequential Organ Failure Assessment (SOFA) score $\geq 2$ (Sepsis-3). It is the result of a dysregulated immune response to infection and one of the leading causes of death in intensive care units [1]. It is estimated that about 30 million patients worldwide are afflicted with sepsis, with potentially over 5 million deaths annually [2]. During the initial phase of sepsis, activated monocytes/macro- phages and neutrophils release a variety of proinflammatory cytokines. The hyperinflammatory response and secondary immunosuppression often lead to multiple organ failure, the major cause of mortality [3].

Early and differential diagnosis of sepsis is essential to decrease morbidity and mortality through prompt antibiotics, vasopressors, and adjuvant therapy [4]. Existing biomarkers for sepsis, such as c-reactive protein (CRP), procalcitonin (PCT), and IL-6, have limited specificity and sensitivity. MicroRNAs (miRNAs) may also serve as diagnostic and prognostic biomarkers in sepsis. miRNAs are short 
noncoding 18- to 25-nucleotide RNAs that bind to the complementary sequence of target mRNAs, mostly in the $3^{\prime}$ untranslated region. They negatively regulate protein expression of target genes via translational repression or mRNA degradation [5]. It has been estimated that up to $30 \%$ of all human genes are miRNA targets, with some miRNAs targeting large numbers of mRNAs [6,7]. Accumulating evidences have demonstrated that miRNAs are regulators of immune response [8]. miRNA-146a and miRNA-146b were shown to target at TRAF6 and IRAK1 and regulated apoptosis of dendritic cells and cytokine production [9]. miR-223 levels were elevated in T cells of patients with rheumatoid arthritis [10]. In addition, miR-155 promoted the survival of Mycobacterium tuberculosis-infected macrophages and favored the survival and function of $\mathrm{T}$ cell immune response [11].

Circulating miRNAs, such as miR-15a, miR-25, miR-34a, miR-133a, miR-146, miR-150, and miR-223, have been described as the biomarkers for sepsis [12-15]. However, the circulating miRNAs are a mixture of miRNAs released extracellularly or contained at apoptotic bodies and exosomes [16]. They may not be ideal biomarkers for early diagnosis. The main sources of inflammatory mediators in sepsis are immune cells. Recently, microRNAs such as miR-10a, miR-17, miR-27a, and miR-125b have been shown to modulate inflammatory response in immune cells. For example, miR-10a was decreased in peripheral blood mononuclear cells (PBMC) from patients of inflammatory bowel diseases and upregulated after treatment with monoclonal antibody for antitumor necrosis factor. miR-10a also inhibited the expression of IL-12/IL-23p40 in human dendritic cells and blocked the Th1/Th17 cell immune response [17]. Lipopolysaccharide (LPS) activated alveolar macrophages by increasing the expression of miR-17, which targeted signal-regulatory protein $\alpha$ and elevated macrophage infiltration, phagocytosis, and secretion of proinflammatory cytokines [18]. In addition, LPS downregulated miR-27a expression in macrophages as a regulatory mechanism to block overly exuberant inflammatory response [19]. In patients with rheumatoid arthritis, miR-125b expression in $\mathrm{CD} 14^{+}$blood monocytes was reduced as compared with healthy controls and negatively correlated with the expression of its target proteins BIK and MTP18, resulting in resistance to oxidative stress and apoptosis [20].

We hypothesized that expression of miRNAs is altered in immune cells during sepsis and might serve as early biomarkers for sepsis. Levels of miR-10a, miR-17, miR-27a, and miR-125b in PBMC were compared among sepsis, infection, and control groups. The results revealed a downregulation of miR-10a in sepsis patients. Downregulation of miR-10a was further validated in a mouse model of cecal ligation and puncture- (CLP-) induced sepsis. Functional experiments in vitro identified an anti-inflammatory role of miR-10a.

\section{Methods}

2.1. Study Subjects. Between January 2018 and May 2018, a total of 41 sepsis patients hospitalized in the ICU of Shaoxing Second Hospital were consecutively enrolled in the study. All sepsis patients fulfilled the definition of the Third International Consensus Definitions for Sepsis and Septic Shock (Sepsis-3, infection + increase $\geq 2$ in SOFA score) [1]. Twenty-one patients admitted in the ICU for infection (infection $+\geq 2$ criteria for systemic inflammatory response syndrome), as a result of pneumonia but without sepsis according to Sepsis-3, were also enrolled. Eligible patients were at least 18 years of age and diagnosed with sepsis or infection without sepsis within the previous $24 \mathrm{~h}$ at any time during their stay in the ICU. Blood samples were collected within 24 hours of admission to the ICU. Twenty healthy volunteers without any signs of infection were enrolled as control (Table 1). Exclusion criteria included cancer, chronic inflammatory diseases, brain injury, $\mathrm{HBV} / \mathrm{HCV} / \mathrm{HIV}$ infection, pregnancy, and refusal of consent. The study was carried out in compliance with the Declaration of Helsinki and was preapproved by the ethics committee of Shaoxing Second Hospital. Written informed consents were obtained from all enrolled patients and healthy volunteers. Demographic and clinical data for infection and septic patients were extracted from the electronic medical record. Patients were observed from ICU admission to day 28 of hospital stay or death.

2.2. Mouse Model of Cecal Ligation and Puncture (CLP). All studies used C57BL/6 male mice (6-8 weeks old; Shanghai Laboratory Animal Center, Shanghai, China). Animal protocols were approved by the animal care committee at Zhejiang University School of Medicine and were in compliance with institutional guidelines. Mice were anesthetized with $4 \%$ trichloroacetaldehyde via intraperitoneal injection. A $2 \mathrm{~cm}$ ventral midline incision was performed to allow the exposure of cecum. The cecum was ligated $1 \mathrm{~cm}$ from the apex with 3-0 silk suture, punctured twice with a 22-gauge needle, and extruded a small amount of fecal material. The abdominal incision was sutured in two layers. Sham surgery was performed with only cecum exposure without ligation and puncture of the cecum in control animals. Isotonic saline solution ( $1 \mathrm{ml} /$ mouse) was administered subcutaneously to all mice to prevent dehydration. Mice were sacrificed at 8 and $24 \mathrm{~h}$ after initial sham or CLP procedure to collect blood for analysis.

\subsection{Total RNA Extraction from Peripheral Blood} Mononuclear Cells. PBMC were separated by density gradient centrifugation using blood samples collected in heparinized tubes. Briefly, blood samples were mixed with an equal volume of phosphate-buffered saline. The mixture was loaded on the top of a Ficoll-Paque density gradient (Cedarlane, Burlington, NC, USA) and pelleted at $2200 \mathrm{rpm}$ for $20 \mathrm{~min}$. PBMC at the interface were harvested and washed twice with phosphate-buffered saline.

2.4. Culture and Treatment of THP-1 Monocytes. THP-1 cells were obtained from the ATCC (Manassas, VA) and maintained in RPMI 1640 medium (Thermo Fisher Scientific) supplemented with $10 \mathrm{U} / \mathrm{ml}$ penicillin-streptomycin, $2 \mathrm{mM}$ L-glutamine, and 10\% fetal bovine serum (Thermo Fisher Scientific) at $37^{\circ} \mathrm{C}$ with $5 \% \mathrm{CO}_{2}$. Cells in log-phase were used for the experiments. THP-1 cells were treated with LPS 
TABLE 1: Demographic and clinical characteristics of sepsis patients.

\begin{tabular}{|c|c|c|c|c|}
\hline Variable & Healthy controls $(n=20)$ & Infection $(n=21)$ & Sepsis $(n=41)$ & $p$ value \\
\hline Age (years) & $65.6 \pm 8.88$ & $72.33 \pm 15.59$ & $74.49 \pm 12.58$ & $>0.05$ \\
\hline Male/female & $11 / 9$ & $11 / 10$ & $28 / 13$ & \\
\hline SOFA score & & $0.48 \pm 0.50$ & $8.65 \pm 3.41$ & $<0.01$ \\
\hline APACHE II score & & & $27.78 \pm 7.66$ & \\
\hline Death $(n)$ & & 0 & 17 & \\
\hline \multicolumn{5}{|l|}{ Microbiology of patients $(n)$} \\
\hline Gram-positive & & 6 & 7 & \\
\hline Gram-negative & & 12 & 18 & \\
\hline No organism cultured & & 3 & 14 & \\
\hline Viral & & 0 & 2 & \\
\hline \multicolumn{5}{|l|}{ Type of infection $(n)$} \\
\hline Pneumonia & & 21 & 31 & \\
\hline Severe cholangitis & & 0 & 2 & \\
\hline Other & & 0 & 8 & \\
\hline $\mathrm{CRP}(\mathrm{mg} / \mathrm{l})$ & & $104.6 \pm 85.52$ & $160.69 \pm 94.2$ & $<0.05$ \\
\hline PCT $(\mathrm{mg} / \mathrm{l})$ & & $2.71 \pm 6.15$ & $8.94 \pm 9.77$ & $<0.01$ \\
\hline
\end{tabular}

Data are presented as patient number or mean \pm SD. APACHE II: Acute Physiology and Chronic Evaluation II; SOFA: Sequential Organ Failure Assessment; CRP: C-reactive protein; PCT: procalcitonin.

(100 ng/ml, E. coli LPS 055:B5, Sigma-Aldrich) for $24 \mathrm{~h}$. After the treatment, cells were harvested for qRT-PCR analysis.

\subsection{Real-Time Quantitative Reverse Transcriptase} Polymerase Chain Reaction ( $q R T-P C R$ ) and miRNA-Specific Primers. Total RNA was isolated from cells using the TRIzol Reagent (Thermo Fisher Scientific). For mRNA analysis, reverse transcription reaction was performed via the PrimeScript $^{\mathrm{TM}}$ RT Reagent Kit (Takara Bio, Kusatsu, Japan) according to the manufacturer's instructions. qRT-PCR was performed using SYBR Green ${ }^{\mathrm{TM}}$ Premix Ex Taq ${ }^{\mathrm{TM}}$ (Takara Bio) on the LightCycler 480 II (Roche). miRNA was determined using the Mix- $\mathrm{X}^{\mathrm{TM}}$ miRNA First Strand Synthesis Kit (Takara Bio) followed by the LightCycler 480 II via the Mir-X miRNA qRT-PCR SYBR Kit (Takara Bio). All target genes were standardized to $\beta$-actin for mRNA or U6 snRNA for miRNA using the standard $\Delta \Delta \mathrm{Ct}$ method. The primer sequences used are listed as follows: IL- 6 forward $5^{\prime} \mathrm{ACTC}$ ACCTCTTCAGAACGAATTG3', reverse $5^{\prime}$ CCATCTTTG

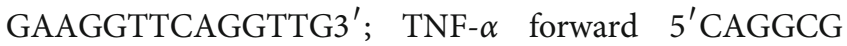
GTGCCTATGTCTC3', reverse $5^{\prime}$ CGATCACCCCGAAG TTCAGTAG3'; MCP-1 forward 5'CAGCCAGATGCAAT CAATGCC $3^{\prime}$, reverse $5^{\prime}$ TGGAATCCTGAACCCACTT $\mathrm{CT}^{\prime}{ }^{\prime} ; \beta$-actin forward $5^{\prime}$ CGTTGACATCCGTAAAGAC $\mathrm{C}^{\prime}{ }^{\prime}$, reverse $5^{\prime}$ AACAGTCCGCCTAGAAGCAC3'; miR10a $5^{\prime}$ CTGTAGATCCGAATTTGTGA3'; miR-27a $5^{\prime}$ AGTGGCTAAGTTCCGCAA3 $^{\prime}$; miR-125b $5^{\prime}$ CCCTGA

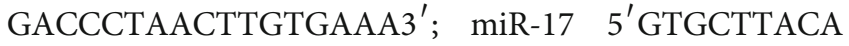
GTGCAGGTAGAA3 $^{\prime}$; and U6 $5^{\prime}$ TCGTGAAGCGTTCC ATATTTTTAA3'.

2.6. Transducing miR-10a into THP-1 Cells. Mouse-miR-10a$5 \mathrm{p}$ and control lentivirus were purchased from Genechem
(Shanghai, China). THP-1 cells were infected with lentiviruses (multiplicity of infection of 20) and cultured for 4 days. THP-1 cells were then stimulated with LPS $(100 \mathrm{ng} / \mathrm{ml})$ for 24 hours. After that, the cells were harvested for qRT-PCR and Western blot analyses.

2.7. Western Blot Analysis. Western blot analysis was performed as described previously [21]. Protein samples were extracted from PBMC of control, infection, and sepsis groups at admission. Equal amounts of protein were loaded on SDSPAGE and transferred to a nitrocellulose membrane. The membrane was probed with a rabbit anti-human mitogenactivated kinase kinase kinase 7 (MAP3K7; TAK1) primary antibody (Cell Signaling Technology, Danvers, MA), followed by incubation with a peroxidase-conjugated goat anti-rabbit secondary antibody (MultiSciences, Hangzhou, China), and detected via enzyme-linked chemiluminescence using the EZ-ECL Kit (Biological Industries, Kibbutz Beit-Haemek, Israel). MAP3K7 levels were normalized to GAPDH expression levels.

2.8. Statistical Analysis. All continuous values with normal distribution were presented as mean \pm standard error of the mean (SEM) and analyzed via Student's $t$-test or an analysis of variance (ANOVA), followed by the Dunn post hoc test for multiple groups. All continuous variables with skewed distribution were expressed as median (interquartile range (IQR)) and analyzed via the Whitney $U$ test. Receiver operating characteristic (ROC) curves and the area under the ROC curves (AUC) were performed to determine the diagnostic and prognostic values of miR-10a. Statistical analysis was carried out using the GraphPad Software (Prism 5.01, GraphPad Software) and SPSS 22.0. Differences were considered as statistically significant if $p<0.05$. 

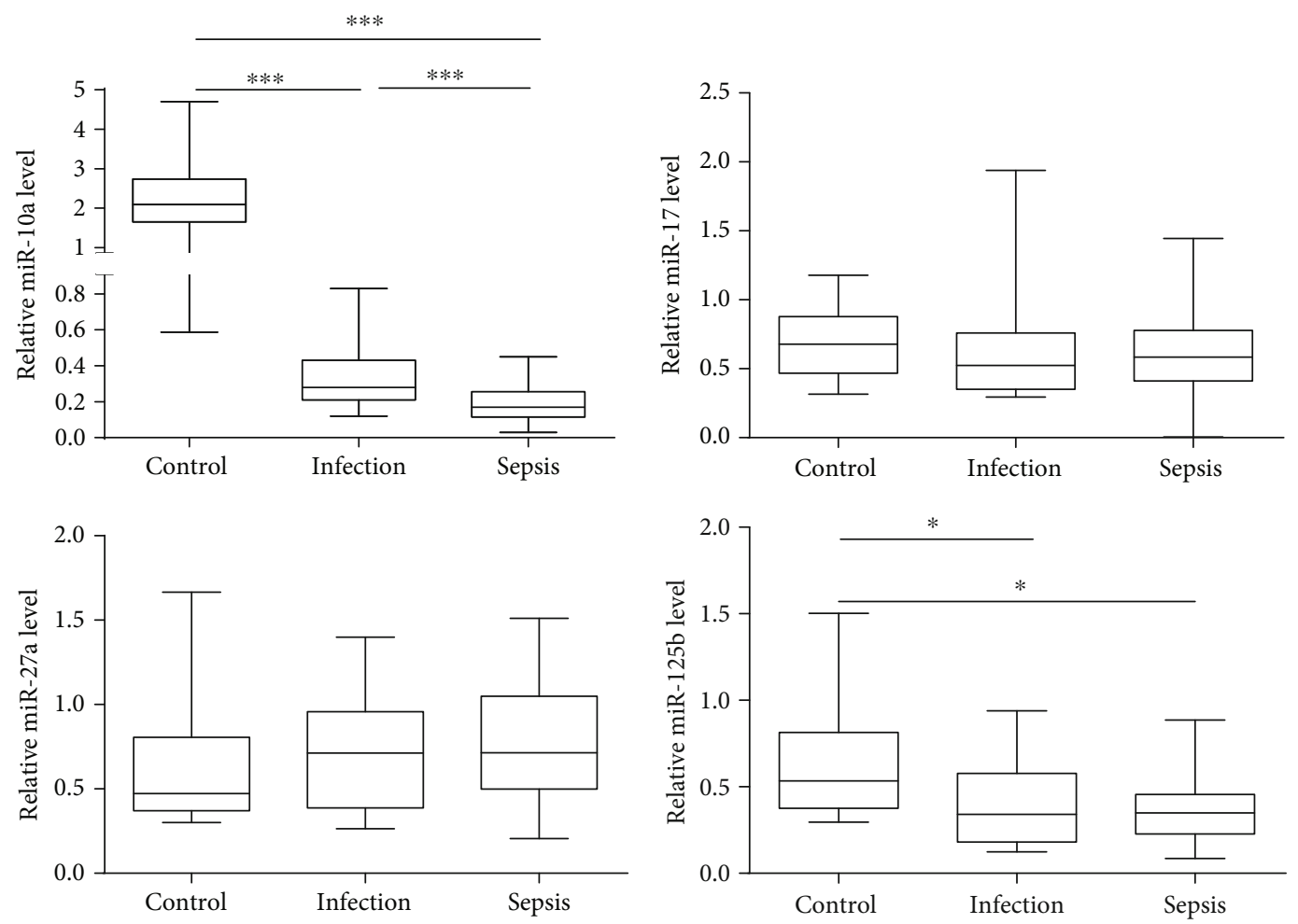

FIgURE 1: PBMC miR-10 levels in sepsis. The box and whisker plots represent the levels of miR-10a, miR-17, miR-27a, and miR-125b in PBMC of healthy controls $(n=20)$, infection patients $(n=21)$, and sepsis patients $(n=41)$. The plot shows the median (lines within boxes), interquartile range (bounds of boxes), and error bars (upper and lower ranges). ${ }^{*} p<0.05$ and ${ }^{* * *} p<0.001$.

\section{Results}

3.1. Study Patient Characteristics. Twenty-one patients with infection, 41 patients with sepsis, and 20 healthy controls were included in the study. The demographic and clinical characteristics of the study patients are shown in Table 1. The major etiologies were pneumonia for both infection and sepsis patients. Sepsis patients had a significantly higher SOFA score and higher CRP and PCT levels than infection patients at enrollment. Patients with infection and sepsis were slightly older than healthy controls, but there was no statistically significant difference in age among the three groups $(p>0.05)$.

3.2. PBMC miR-10a Levels Are Reduced in Sepsis Patients. To explore the potential value of PBMC miRNAs as a biomarker for sepsis, levels of 4 inflammation-related miRNAs (miR10a, miR-17, miR-27a, and miR-125b) in PBMC at admission were examined in 41 patients with sepsis, 21 patients with infection, and 20 healthy controls via qRT-PCR (Figure 1). miR-10a levels in sepsis patients were lower than those in infection patients and healthy controls. Infection patients also showed reduced levels of miR-10a compared with healthy controls. There was no difference in miR-17 and miR-27a levels among the control, infection, and sepsis patients. miR-125b expression was decreased in sepsis and infection patients compared with healthy controls. However, there was no difference in $\mathrm{miR}-125 \mathrm{~b}$ expression between sep- sis and infection patients, indicating that miR-125b could not differentiate sepsis from infection.

3.3. miR-10a Levels in PBMC from Sepsis Patients Correlate with Disease Severity Scores and Existing Biomarkers for Sepsis. To elucidate the clinical relevance of miR-10a in sepsis, Spearman's correlation analysis was conducted between levels of miR-10a in PBMC and the disease severity scoring systems using the Acute Physiology and Chronic Evaluation II (APACHE II) and the SOFA. Strong negative linear relationships existed between levels of miR$10 \mathrm{a}$ and APACHE II scores $(r=-0.331, p=0.034)$ as well as SOFA scores $(r=-0.368, p=0.018)$ (Figure 2(a)). In addition, miR-10a levels in PBMC were also significantly correlated to levels of CRP $(r=-0.418, p=0.007)$ and PCT $(r=-0.323, p=0.039)$, two popular biomarkers of sepsis (Figure 2(b)).

3.4. PBMC miR-10a Has Diagnostic Performance for Sepsis. ROC curve analysis was applied to compare the diagnostic value of PBMC miR-10a, CRP, and PCT between infection and sepsis. The AUC for miR-10a (AUC $=0.804 ; 95 \% \mathrm{CI}$, $0.688-0.920 ; p<0.001)$ was comparable to that of PCT $(\mathrm{AUC}=0.792 ; 95 \% \mathrm{CI}, 0.664-0.920 ; p<0.001)$ and better than the diagnostic performance of CRP (AUC $=0.669$; 95\% CI, 0.518-0.820; $p<0.01$ ) (Figure 3). These findings suggest that PBMC miR-10a could distinguish between sepsis and infection and could perform at least as well as PCT and CRP for sepsis diagnosis. When the cut-off point for miR- 

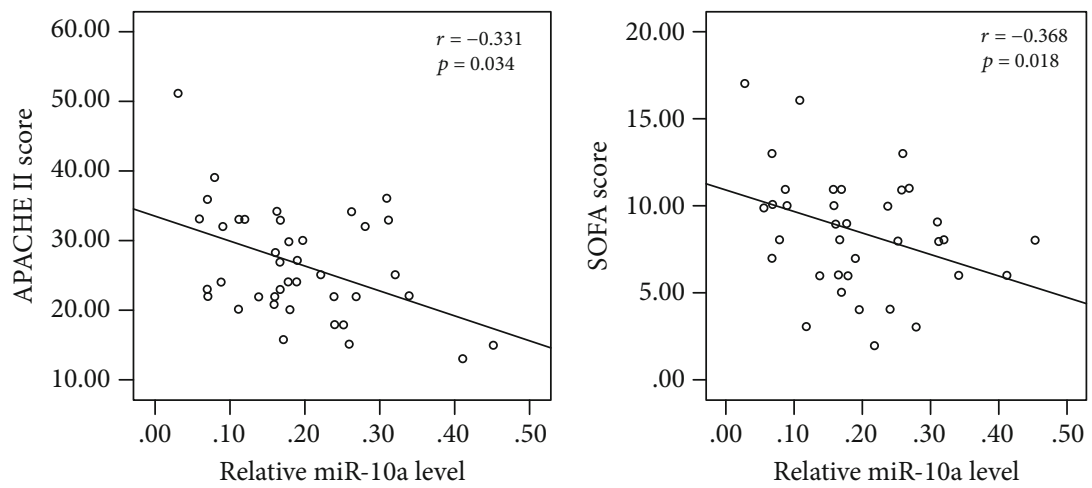

(a)
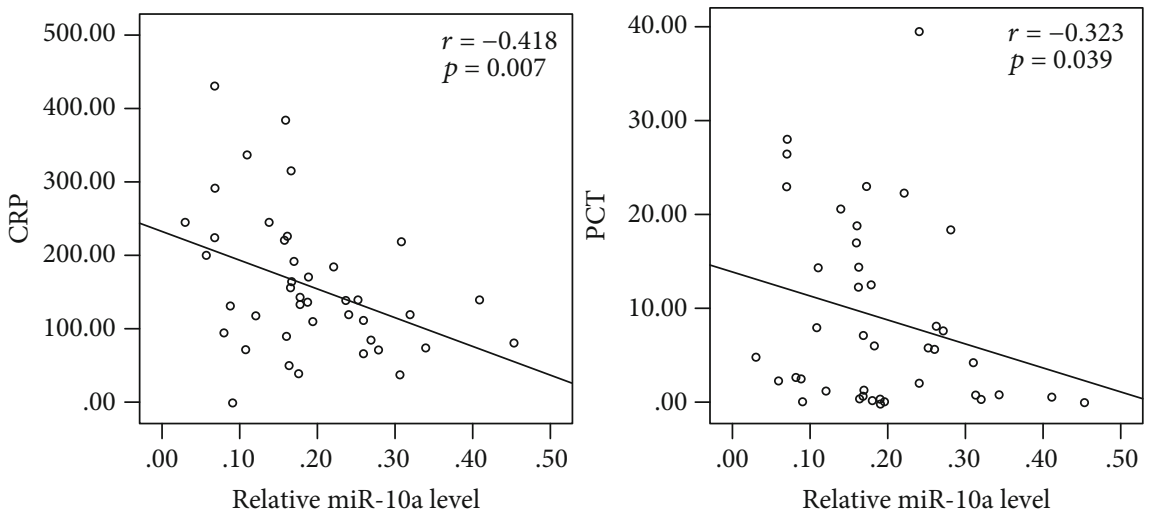

(b)

Figure 2: Correlation analysis of PBMC miR-10a levels with disease severity scores and sepsis biomarkers. (a) The curve was plotted by miR10 a relative expression values at admission of 41 sepsis patients to their respective APACHE II and SOFA scores. (b) The curve was plotted by miR-10a relative expression levels at admission to their respective CRP and PCT values. Each circle represents an individual patient.

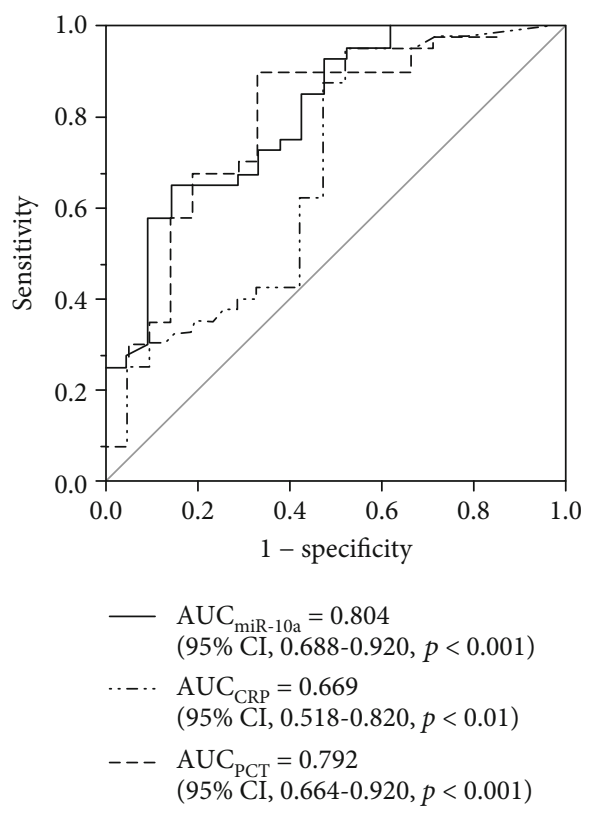

FIGURE 3: ROC curves for the prediction of sepsis. ROC curves present sensitivity and specificity of PBMC miR-10a, CRP, and PCT for predicting sepsis.
$10 \mathrm{a}$ was set at 0.18 , it had a sensitivity of $65.0 \%$ and a specificity of $85.7 \%$.

3.5. PBMC miR-10a Has Predictive Value for 28-Day Mortality. All enrolled patients were monitored for 28 days after enrollment or until death. PBMC miR-10a levels at admission were significantly lower in nonsurvivors $(n=17)$ than in survivors $(n=45)(p<0.05)$ (Figure 4(a)). The performance of miR-10a, PCT, and CRP for predicting 28-day mortality was evaluated by ROC curve analysis between hospital deaths and survivors for all enrolled patients. PCT performed the best for predicting 28-day mortality $(\mathrm{AUC}=0.795 ; 95 \% \mathrm{CI}, 0.664-0.920 ; p<0.05)$. PBMC miR10a $(\mathrm{AUC}=0.699 ; 95 \% \mathrm{CI}, 0.656-0.833 ; p<0.05)$ displayed a similar prognostic performance as CRP (AUC $=0.705$; 95\% CI, 0.568-0.841; $p<0.001$ ) (Figure 4(b)).

3.6. PBMC MAP3K7 Levels at Admission Are Higher in Sepsis Patients. It has been reported that miR-10a targets MAP3K7 and IRAK4, which act upstream of NF- $\kappa$ B activation [22]. To determine whether altered miR-10a expression affected the level of MAP3K7 and IRAK4 in sepsis, PBMC from sepsis patients, infection patients, and healthy controls at admission were examined via Western blot analysis. Levels of MAP3K7 from sepsis patients were significantly higher than those of infection patients and healthy controls. In addition, 


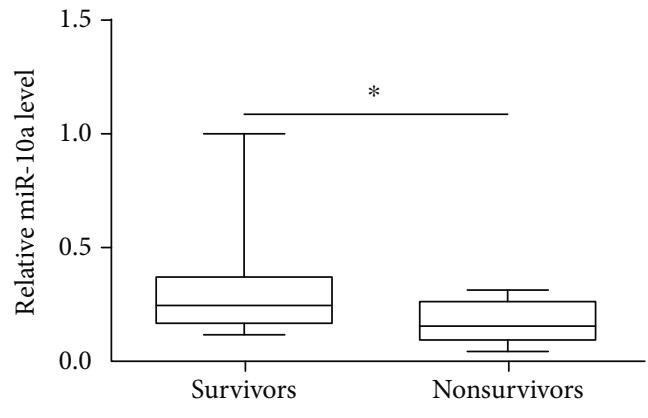

(a)

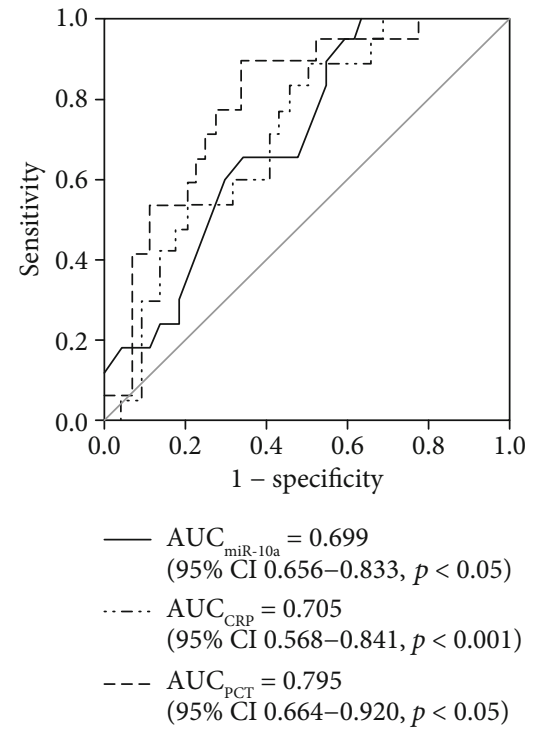

(b)

FIgURE 4: ROC curves for the prediction of 28-day mortality. (a) PBMC miR-10a levels were compared between nonsurvivors $(n=17)$ and survivors $(n=45)$. (b) ROC curves present sensitivity and specificity of PBMC miR-10a, CRP, and PCT for predicting 28-day mortality.

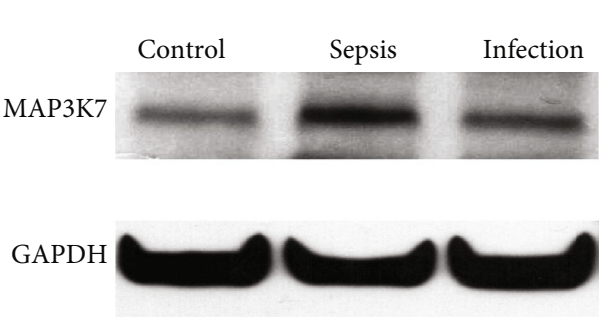

(a)

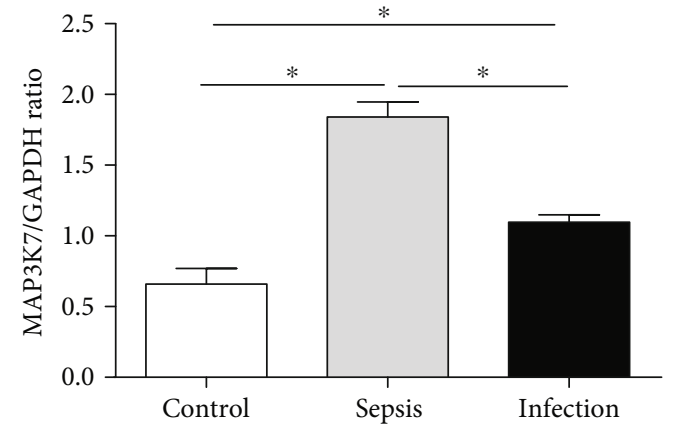

(b)

FIGURE 5: MAP3K7 expression in PBMC of sepsis patients with respect to infection and control. PBMC were collected from sepsis patients, infection patients, and healthy controls at admission. MAP3K7 protein expression in PBMC was examined via Western blot analysis. (a) A representative Western blot is shown. (b) Data are presented as mean $\pm \mathrm{SEM}, n=6 .{ }^{*} p<0.05$.

MAP3K7 expression in infection patients was higher than that of control (Figures 5(a) and 5(b)). However, there was no difference in IRAK4 levels among the three groups (data not shown). These findings suggest that MAP3K7 is a target of miR-10a in immune cells.

3.7. Polymicrobial Sepsis Decreases the Levels of miR-10a in $P B M C$. To determine the effects of polymicrobial sepsis on the expression of miR-10a, sepsis was induced in a mouse model by CLP. PBMC were harvested at $8 \mathrm{~h}$ and $24 \mathrm{~h}$ after CLP and examined for miR-10a levels via qRT-PCR. As shown in Figure 6(a), polymicrobial sepsis significantly decreased the expression of miR-10a as early as $8 \mathrm{~h}$ after CLP surgery compared with sham operation $(p<0.01)$. PBMC miR-10a levels remained significantly diminished at $24 \mathrm{~h}$ after surgery $(p<0.05)$ (Figure 6(b)). These data demonstrate that miR-10a expression is reduced by polymicrobial sepsis and may serve as an early biomarker for sepsis.
3.8. miR-10a Overexpression Suppresses LPS-Induced Inflammatory Response In Vitro. We then asked whether overexpression of miR-10a could modulate LPS-induced inflammatory response in vitro. THP-1 monocytes expressed high levels of miR-10a after having been transduced with lentivirus expressing miR-10a (Figure 7(a)). miR-10atransduced cells had lower expression of MAP3K7, further supporting that MAP3K7 is a target of miR-10a (Figure 7(b)). In addition, miR-10a overexpression suppressed the mRNA expression of IL-6, TNF- $\alpha$, and MCP-1 induced by LPS as determined by qRT-PCR (Figure $7(\mathrm{c})$ ). These data suggest that miR-10a is a negative regulator for proinflammatory cytokines induced by LPS.

\section{Discussion}

To the best of our knowledge, this is the first report demonstrating that PBMC miRNAs could serve as biomarkers for 


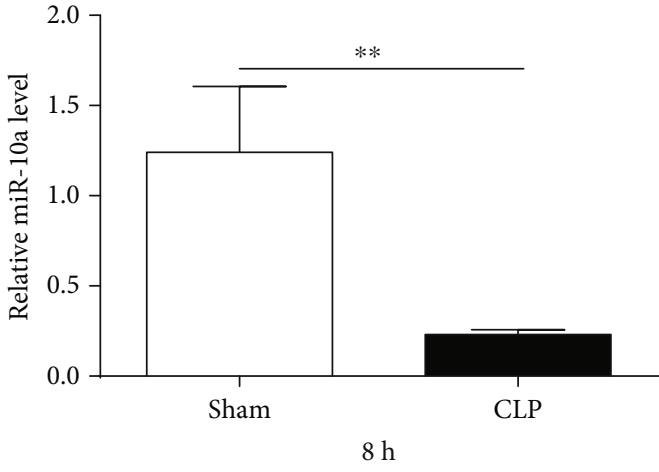

(a)

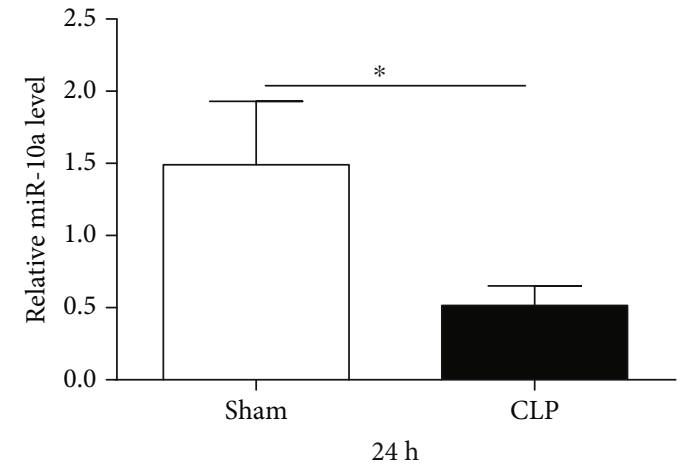

(b)

FIGURE 6: miR-10a expression in a mouse model of sepsis. PBMC were isolated from whole blood of C57BL/6 mice after $8 \mathrm{~h}$ (a) and $24 \mathrm{~h}$ (b) of sham or CLP surgery. miR-10a expression was determined via qRT-PCR. Data are presented as mean \pm SEM, $n=6 .{ }^{*} p<0.05$ and ${ }^{* *} p<0.01$.

the diagnosis and prognosis of sepsis. Our results showed that sepsis patients presented significantly reduced expression of miR-10a compared with both infection patients and healthy controls. There was a significantly negative correlation between miR-10a levels and disease severity scores. In addition, PBMC miR-10a was at least as good as PCT and $\mathrm{CRP}$ in differentiating sepsis and infection. PBMC miR-10a levels were significantly lower in nonsurvivors than in survivors and predictive of 28-day mortality. Reduced miR-10a expression was also validated in a mouse model of polymicrobial sepsis. Furthermore, overexpression of miR-10a in THP-1 cells reduced the expression of MAP3K7 and proinflammatory cytokines induced by LPS, demonstrating the anti-inflammatory effect of miR-10a.

Sepsis-3 defines sepsis as life-threatening organ dysfunction resulting from dysregulated host response to infection. An inflammatory response which is part of a normal response to infection does not necessarily indicate sepsis. Early diagnosis of sepsis relies on laboratory biomarkers and clinical scores. However, most common biomarkers for sepsis diagnosis including PCT, CRP, and interleukin 6 have limited specificity and sensitivity. Our study demonstrated that levels of PBMC miR-10a at admission were significantly lower in sepsis patients than in infection patients and negatively associated with disease severity scores. In addition, PBMC miR-10a could distinguish sepsis from infection in our study population (AUC $=0.804 ; 95 \% \mathrm{CI}, 0.688-0.920 ; p$ $<0.001)$. These findings provide evidences that PBMC miR-10a has the potential to be a novel diagnostic marker for sepsis. Furthermore, low PBMC miR-10a levels predicted an unfavorable short-term outcome, suggesting the role of PBMC miR-10a in the prognosis of sepsis. Given the complex pathophysiology of sepsis, no single biomarker of sepsis may be ideal for diagnosis. The application of multiple biomarkers has been reported to provide improved diagnostic accuracy to differentiate sepsis from infection [23]. Therefore, the combination of PBMC miR-10a with traditional biomarkers may be applied for the accurate diagnosis of sepsis.

During the past 10 years, circulating miRNAs have been explored as new biomarkers for sepsis. Analysis of miRNAs has the advantage of earlier detection compared to protein biomarkers since protein expression is located downstream of miRNA function. In addition, miRNA expression may enhance risk stratification and serve as a useful prognostic biomarker for sepsis. Such tool could provide guidance for early and aggressive intervention. However, circulating miRNAs are not directly related to cytokine-producing immune cells and may not be the earliest biomarkers for sepsis. Almost all cells exposed to serum are able to produce circulating miRNAs including neutrophils, platelets, lymphocytes, monocytes, endothelial cells, and endothelial progenitor cells $[24,25]$. Secondly, a study in circulating miRNAs could not pinpoint the direct target proteins of the miRNAs in vivo and examine the detailed molecular mechanisms downstream of the miRNAs. Therefore, we evaluated the application of PBMC miRNAs as biomarkers for sepsis since $\mathrm{PBMC}$ are a major contributor of inflammatory cytokines during sepsis. Accumulating evidences support the application of PBMC miRNAs as disease biomarkers. Winger et al. reported that PBMC miRNA levels in the early first trimester predicted adverse outcomes such as miscarriage and late preeclampsia [26]. In treated HIV-1-infected individuals, Ballegaard et al. found that miR-210, miR-7, and miR-331 in PBMC were differentially expressed and correlated with biomarkers of systemic inflammation [27]. Dong et al. revealed that elevated expression of miR-24, miR-33a, miR-103a, and miR-122 in PBMC was associated with increased risk of coronary artery disease [28].

miR-10a has an anti-inflammatory effect and plays an essential role in the inflammatory response of immune cells [29]. Takahashi et al. documented that TGF- $\beta$ and retinoic acid induced the expression of miR-10a, which constrained the differentiation of Th17 cells and inhibited the production of proinflammatory IL-17A [30]. Wu et al. discovered that miR-10a inhibited mucosal inflammatory response via targeting IL-12/IL-23p40 and NOD2 and blocking the immune response of Th1/Th17 cells [17]. Mu et al. showed that miR10a levels were reduced in the fibroblast-like synoviocytes of rheumatoid arthritis patients compared with osteoarthritis controls [31]. It has been demonstrated that miR-10a could exert anti-inflammatory effects by targeting several members of the NF- $\kappa$ B pathway. Njock et al. found that inhibition of miR-10a in monocytes abolished the inhibitory effects of 

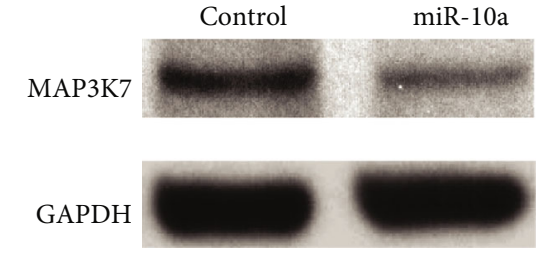

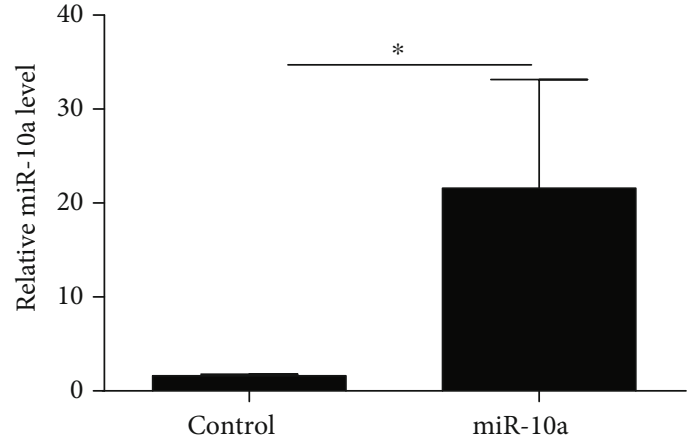

(a)
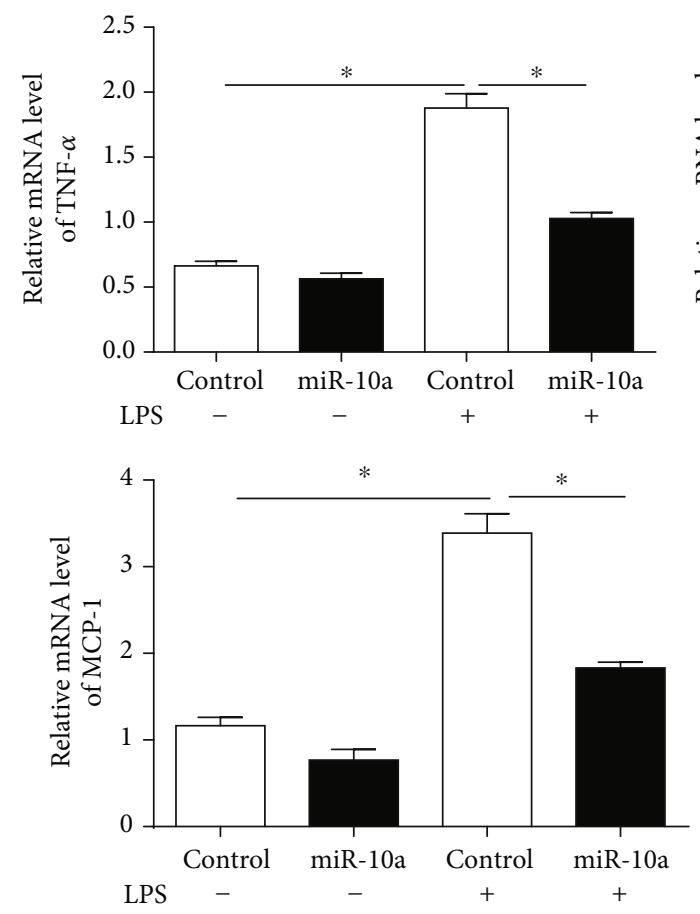

(c)

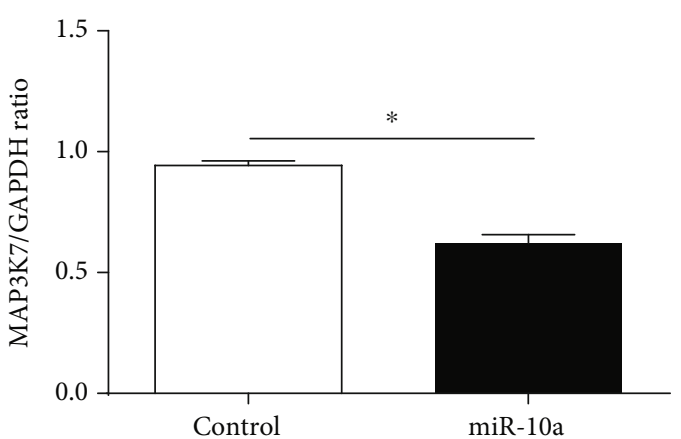

(b)
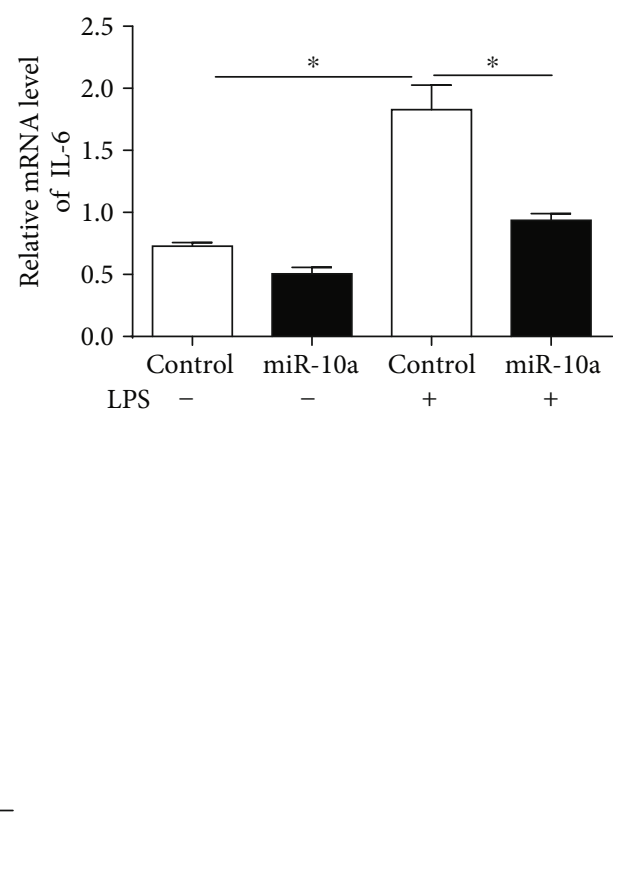

FiguRE 7: Effect of miR-10a overexpression on LPS-induced inflammatory cytokine production in monocytes. THP-1 monocytes were transduced with lentiviral miR-10a or miR-control. (a) Four days after the transduction, miR-10a levels in THP-1 cells were determined via qRT-PCR. (b) MAP3K7 expression in transduced THP-1 cells was determined via Western blot analysis. (c) The transduced cells were treated with or without LPS $(100 \mathrm{ng} / \mathrm{ml})$ for $24 \mathrm{~h}$. Levels of IL-6, TNF- $\alpha$, and MCP-1 in treated cells were assayed via qRT-PCR. Data are presented as mean \pm SEM, $n=4 .{ }^{*} p<0.05$.

endothelial cell-derived extracellular vesicles on proinflammatory genes via upregulating IRAK4 [32]. Fang et al. discovered that endothelial miR-10a levels were lower in the atherosusceptible regions than in the atheroresistant regions in the pig. The endothelium in atherosusceptible regions had upregulated the expression of MAP3K7 and BTRC as well as NF- $\kappa$ B activation [22]. In addition, the induction of miRNA$10 \mathrm{a}$ via retinoic acid receptor- $\alpha$ or retinoid $\times$ receptor $-\alpha$ agonists blocked the formation of an atherosclerotic lesion in rats [33]. In the present study, MAP3K7 levels in PBMC were elevated in sepsis patients and diminished in THP-1 cells overexpressing miR-10a, supporting that MAP3K7 is a target of miR-10a in immune cells.

Our study has several limitations. First, the sample size is small, although a small number of patients have been used in similar studies [34]. Further investigation is warranted to 
confirm the present findings. Our report is also limited by studying a single time point at admission in a disease with a prolonged course and complex cellular mechanisms. A time course study of PBMC miR-10a expression is warranted to confirm the utility of miR-10a as a biomarker for sepsis. Furthermore, it has been documented that miR-10a is expressed by all immune cell types in PBMC including T cells, B cells, natural killer cells, dendritic cells, and monocytes [17, 35]. This study is still incapable of concluding the cell type(s) contributing to the reduced expression of miR-10a in sepsis.

\section{Conclusions}

In summary, miR-10a has an anti-inflammatory capacity during immune response to sepsis. PBMC miR-10a levels correlate with the disease severity of sepsis. Importantly, low PBMC miR-10a expression is a diagnostic biomarker for sepsis and predictive of 28-day mortality. Due to the small sample size of the present study, a larger multicenter study will determine the value of a miR-10a-based biomarker for sepsis diagnosis and prognosis.

\section{Abbreviations}

PBMC: $\quad$ Peripheral blood mononuclear cells

CLP: $\quad$ Cecal ligation and puncture

MAP3K7: $\quad$ Mitogen-activated kinase kinase kinase 7

qRT-PCR: Real-time quantitative reverse transcriptase polymerase chain reaction

APACHE II: Acute Physiology and Chronic Evaluation II

SOFA: Sequential Organ Failure Assessment

CRP: $\quad$ C-reactive protein

PCT: Procalcitonin

ICU: Intensive care unit

ROC: $\quad$ Receiver operating characteristic

IQR: Interquartile range

AUC: $\quad$ Area under the curve.

\section{Data Availability}

The dataset supporting the conclusions of this article is included within the article.

\section{Ethical Approval}

The study was approved by the ethics committee of Shaoxing Second Hospital and was conducted in accordance with the Declaration of Helsinki.

\section{Consent}

All patients or their relatives provided informed consent to participate.

\section{Conflicts of Interest}

All authors declare that they have no potential conflicts of interest.

\section{Authors' Contributions}

GZ and GQ participated in the study design, patient recruitment, data collection, data analysis, and manuscript preparation. MG, JW, and RH contributed in patient recruitment, data collection, and manuscript preparation. JM and GZ contributed in data collection and in the editing of the manuscript. QS and JX participated in the study design, data analysis, interpretation of results, and editing of the manuscript. All authors read and approved the final manuscript. Guoping Zheng and Guanguan Qiu contributed equally to this work.

\section{Acknowledgments}

The authors thank the medical and nursing staff at the ICU department of Shaoxing Second Hospital. This work was supported by the Natural Science Foundation of Zhejiang Province (Grant Nos. LYY18H310008 and LGF18H150007), the Health Commission of Zhejiang Province (Grant Nos. 2020KY335 and 2020KY992), the Zhejiang Provincial Program for the Cultivation of High-Level Innovative Health Talents (Grant No. 2016-6), and the National Scientific Foundation of China (Grant Nos. 81671956, 81570071, and 81772122).

\section{References}

[1] M. Singer, C. S. Deutschman, C. W. Seymour et al., "The third international consensus definitions for sepsis and septic shock (Sepsis-3)," JAMA, vol. 315, no. 8, pp. 801-810, 2016.

[2] C. Fleischmann, A. Scherag, N. K. J. Adhikari et al., "Assessment of global incidence and mortality of hospital-treated sepsis. current estimates and limitations," American Journal of Respiratory and Critical Care Medicine, vol. 193, no. 3, pp. 259-272, 2016.

[3] J. S. Boomer, J. M. Green, and R. S. Hotchkiss, "The changing immune system in sepsis: is individualized immunomodulatory therapy the answer?," Virulence, vol. 5, no. 1, pp. 45-56, 2014.

[4] P. E. Marik and J. D. Farkas, "The changing paradigm of sepsis: early diagnosis, early antibiotics, early pressors, and early adjuvant treatment," Critical Care Medicine, vol. 46, no. 10, pp. 1690-1692, 2018.

[5] D. P. Bartel, "Metazoan microRNAs," Cell, vol. 173, no. 1, pp. 20-51, 2018.

[6] B. P. Lewis, C. B. Burge, and D. P. Bartel, "Conserved seed pairing, often flanked by adenosines, indicates that thousands of human genes are microRNA targets," Cell, vol. 120, no. 1, pp. 15-20, 2005.

[7] L. P. Lim, N. C. Lau, P. Garrett-Engele et al., "Microarray analysis shows that some microRNAs downregulate large numbers of target mRNAs," Nature, vol. 433, no. 7027, pp. 769-773, 2005.

[8] T. S. Cohen, "Role of microRNA in the lung's innate immune response," Journal of Innate Immunity, vol. 9, no. 3, pp. 243249, 2017.

[9] H. Park, X. Huang, C. Lu, M. S. Cairo, and X. Zhou, "MicroRNA-146a and microRNA-146b regulate human dendritic cell apoptosis and cytokine production by targeting TRAF6 and 
IRAK1 proteins," The Journal of Biological Chemistry, vol. 290, no. 5, pp. 2831-2841, 2015.

[10] V. Fulci, G. Scappucci, G. D. Sebastiani et al., "miR-223 is overexpressed in T-lymphocytes of patients affected by rheumatoid arthritis," Human Immunology, vol. 71, no. 2, pp. 206-211, 2010.

[11] A. C. Rothchild, J. R. Sissons, S. Shafiani et al., "MiR-155-regulated molecular network orchestrates cell fate in the innate and adaptive immune response to Mycobacterium tuberculosis," Proceedings of the National Academy of Sciences of the United States of America, vol. 113, no. 41, pp. E6172-E6181, 2016.

[12] D. E. Giza, E. Fuentes-Mattei, M. D. Bullock et al., "Cellular and viral microRNAs in sepsis: mechanisms of action and clinical applications," Cell Death and Differentiation, vol. 23, no. 12, pp. 1906-1918, 2016.

[13] C. Roderburg, M. Luedde, D. Vargas Cardenas et al., "Circulating microRNA-150 serum levels predict survival in patients with critical illness and sepsis," PLoS One, vol. 8, no. 1, article e54612, 2013.

[14] F. Tacke, C. Roderburg, F. Benz et al., "Levels of circulating miR-133a are elevated in sepsis and predict mortality in critically ill patients," Critical Care Medicine, vol. 42, no. 5, pp. 1096-1104, 2014.

[15] A. J. Goodwin, C. Guo, J. A. Cook, B. Wolf, P. V. Halushka, and H. Fan, "Plasma levels of microRNA are altered with the development of shock in human sepsis: an observational study," Critical Care, vol. 19, no. 1, 2015.

[16] J. M. Real, L. R. P. Ferreira, G. H. Esteves et al., "Exosomes from patients with septic shock convey miRNAs related to inflammation and cell cycle regulation: new signaling pathways in sepsis?," Critical Care, vol. 22, no. 1, p. 68, 2018.

[17] W. Wu, C. He, C. Liu et al., "miR-10a inhibits dendritic cell activation and Th1/Th17 cell immune responses in IBD," Gut, vol. 64, no. 11, pp. 1755-1764, 2015.

[18] D. Zhu, C. Pan, L. Li et al., "MicroRNA-17/20a/106a modulate macrophage inflammatory responses through targeting signalregulatory protein $\alpha$," The Journal of Allergy and Clinical Immunology, vol. 132, no. 2, pp. 426-436.e8, 2013.

[19] N. Xie, H. Cui, S. Banerjee et al., "miR-27a regulates inflammatory response of macrophages by targeting IL-10," Journal of Immunology, vol. 193, no. 1, pp. 327-334, 2014.

[20] I. Duroux-Richard, C. Roubert, M. Ammari et al., "miR-125b controls monocyte adaptation to inflammation through mitochondrial metabolism and dynamics," Blood, vol. 128, no. 26, pp. 3125-3136, 2016.

[21] G. Qiu, G. Zheng, M. Ge et al., "Adipose-derived mesenchymal stem cells modulate $\mathrm{CD} 14^{++} \mathrm{CD} 16^{+}$expression on monocytes from sepsis patients in vitro via prostaglandin E2," Stem Cell Research \& Therapy, vol. 8, no. 1, p. 97, 2017.

[22] Y. Fang, C. Shi, E. Manduchi, M. Civelek, and P. F. Davies, "MicroRNA-10a regulation of proinflammatory phenotype in athero-susceptible endothelium in vivo and in vitro," Proceedings of the National Academy of Sciences of the United States of America, vol. 107, no. 30, pp. 13450-13455, 2010.

[23] H. H. Dolin, T. J. Papadimos, S. Stepkowski, X. Chen, and Z. K. Pan, "A novel combination of biomarkers to herald the onset of sepsis prior to the manifestation of symptoms," Shock, vol. 49, no. 4, pp. 364-370, 2018.

[24] Y. Pan, H. Liang, H. Liu et al., "Platelet-secreted microRNA223 promotes endothelial cell apoptosis induced by advanced glycation end products via targeting the insulin-like growth factor 1 receptor," Journal of Immunology, vol. 192, no. 1, pp. 437-446, 2014.

[25] M. Mittelbrunn, C. Gutiérrez-Vázquez, C. Villarroya-Beltri et al., "Unidirectional transfer of microRNA-loaded exosomes from T cells to antigen-presenting cells," Nature Communications, vol. 2, no. 1, 2011.

[26] E. E. Winger, J. L. Reed, and X. Ji, "First trimester PBMC microRNA predicts adverse pregnancy outcome," American Journal of Reproductive Immunology, vol. 72, no. 5, pp. 515526, 2014.

[27] V. Ballegaard, U. Ralfkiaer, K. K. Pedersen et al., "MicroRNA210, microRNA-331, and microRNA-7 are differentially regulated in treated HIV-1-infected individuals and are associated with markers of systemic inflammation," Journal of Acquired Immune Deficiency Syndromes, vol. 74, no. 4, pp. e104-e113, 2017.

[28] J. Dong, Y. Z. Liang, J. Zhang et al., "Potential role of lipometabolism-related microRNAs in peripheral blood mononuclear cells as biomarkers for coronary artery disease," Journal of Atherosclerosis and Thrombosis, vol. 24, no. 4, pp. 430-441, 2017.

[29] N. Moradi, R. Fadaei, R. Ahmadi, E. Kazemian, and S. Fallah, "Lower expression of miR-10a in coronary artery disease and its association with pro/anti-inflammatory cytokines," Clinical Laboratory, vol. 64, no. 5, pp. 847-854, 2018.

[30] H. Takahashi, T. Kanno, S. Nakayamada et al., "TGF- $\beta$ and retinoic acid induce the microRNA miR-10a, which targets Bcl-6 and constrains the plasticity of helper T cells," Nature Immunology, vol. 13, no. 6, pp. 587-595, 2012.

[31] N. Mu, J. Gu, T. Huang et al., "A novel NF- $\kappa B / Y Y 1 / m i-$ croRNA-10a regulatory circuit in fibroblast-like synoviocytes regulates inflammation in rheumatoid arthritis," Scientific Reports, vol. 6, no. 1, article 20059, 2016.

[32] M. S. Njock, H. S. Cheng, L. T. Dang et al., "Endothelial cells suppress monocyte activation through secretion of extracellular vesicles containing antiinflammatory microRNAs," Blood, vol. 125, no. 20, pp. 3202-3212, 2015.

[33] D. Y. Lee, T. L. Yang, Y. H. Huang et al., "Induction of microRNA-10a using retinoic acid receptor- $\alpha$ and retinoid $\mathrm{x}$ receptor- $\alpha$ agonists inhibits atherosclerotic lesion formation," Atherosclerosis, vol. 271, pp. 36-44, 2018.

[34] H. Brodska, J. Valenta, K. Pelinkova et al., "Diagnostic and prognostic value of presepsin vs. established biomarkers in critically ill patients with sepsis or systemic inflammatory response syndrome," Clinical Chemistry and Laboratory Medicine, vol. 56, no. 4, pp. 658-668, 2018.

[35] H. Huang, L. Fan, R. Zhan, S. Wu, and W. Niu, "Expression of microRNA-10a, microRNA-342-3p and their predicted target gene TIAM1 in extranodal NK/T-cell lymphoma, nasal type," Oncology Letters, vol. 11, no. 1, pp. 345-351, 2016. 


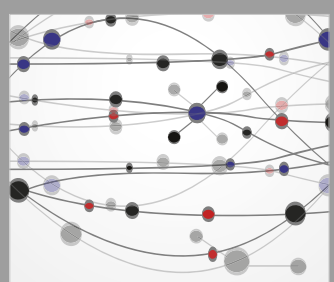

The Scientific World Journal
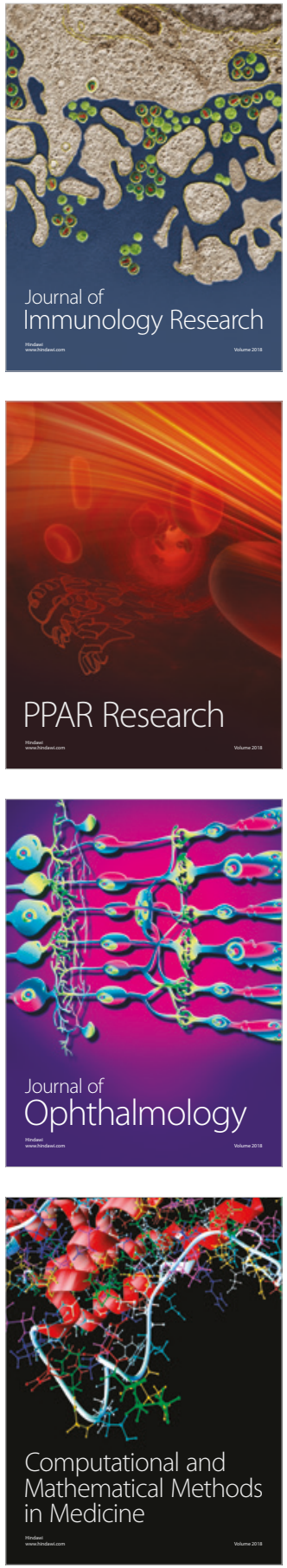

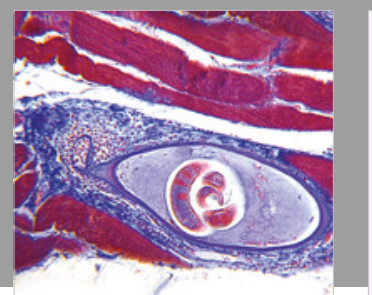

Gastroenterology Research and Practice

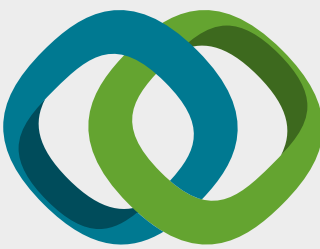

\section{Hindawi}

Submit your manuscripts at

www.hindawi.com
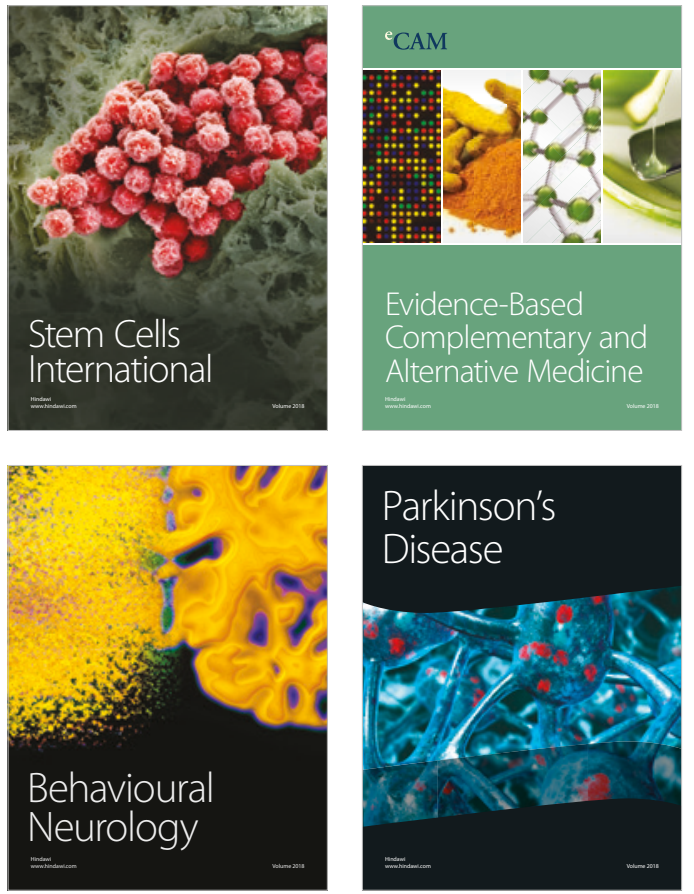

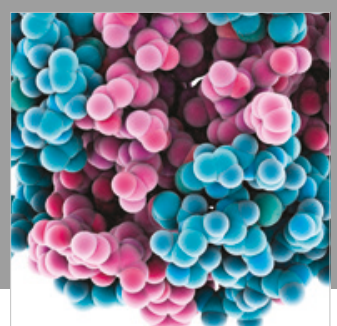

ournal of

Diabetes Research

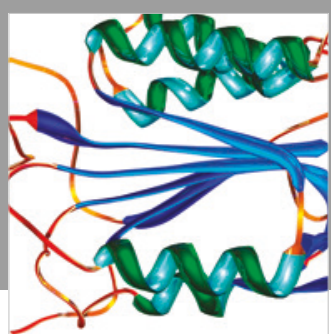

Disease Markers
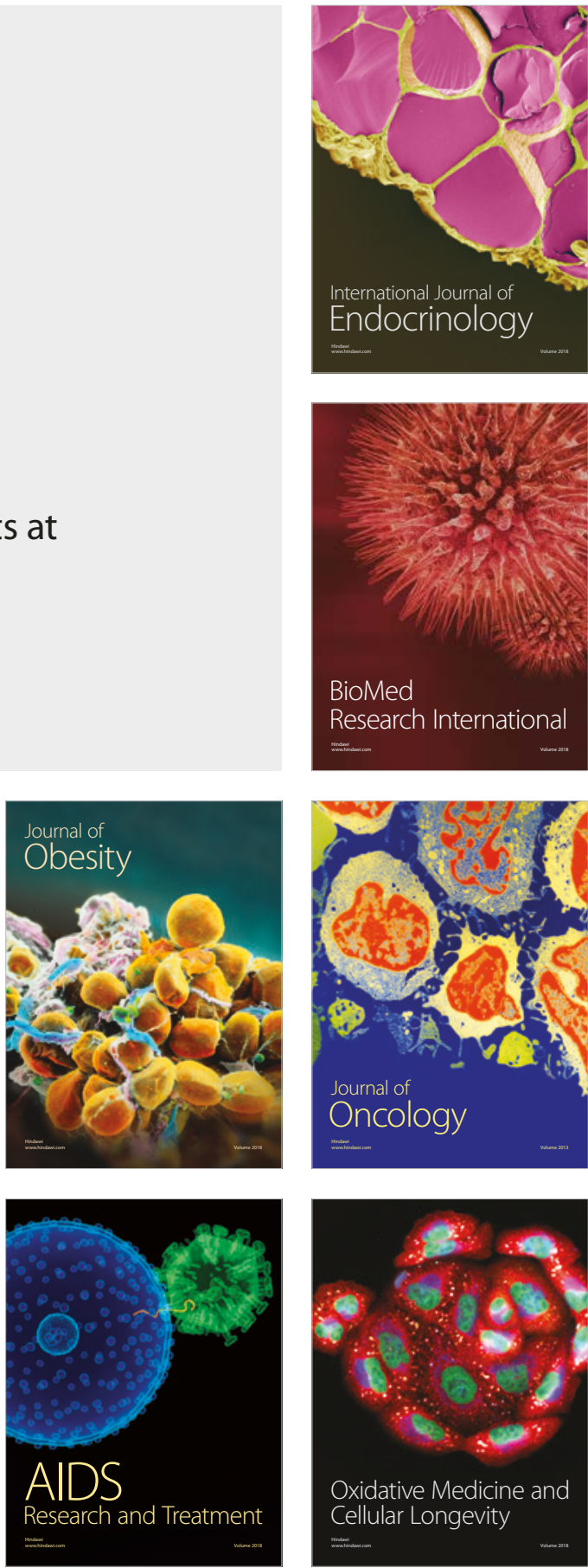